Xavier Roulleau

\title{
Elliptic curve configurations on Fano surfaces
}

Received: 5 May 2008 / Revised: 11 February 2009

Published online: 1 April 2009

\begin{abstract}
The elliptic curves on a surface of general type constitute an obstruction for the cotangent sheaf to be ample. In this paper, we give the classification of the configurations of the elliptic curves on the Fano surface of a smooth cubic threefold. That means that we give the number of such curves, their intersections and a plane model. This classification is linked to the classification of the automorphism groups of theses surfaces.
\end{abstract}

\section{Introduction}

Considering a variety $S$, it is a natural question to study the ampleness of its cotangent sheaf. Recall that, by definition, a bundle $\mathcal{E}$ on $S$ is ample if the tautological sheaf $\mathcal{O}_{\mathbb{P}\left(\mathcal{E}^{*}\right)}(1)$ is an ample line bundle of the projective bundle $\mathbb{P}\left(\mathcal{E}^{*}\right)$ of one dimensional sub-spaces of $\mathcal{E}^{*}$. Gieseker gives the following criteria of ampleness if in addition the bundle $\mathcal{E}$ is generated by its space of global sections:

Proposition 1. (Gieseker [8]) The bundle $\mathcal{E}$ is ample if and only if for every curve $C \hookrightarrow S$, the bundle $\mathcal{E} \otimes \mathcal{O}_{C}$ has no quotient isomorphic to the trivial sheaf $\mathcal{O}_{C}$.

Hence, we consider varieties with the following assumptions:

Hypothesis 2. The variety $S$ is a smooth complex surface of general type. The cotangent sheaf $\Omega_{S}$ of $S$ is generated by its global sections and the irregularity $q=\operatorname{dim} H^{0}\left(\Omega_{S}\right)$ satisfies $q>3$.

With the criteria of Gieseker in mind, a curve $C \hookrightarrow S$ is called non-ample if and only if the sheaf $\Omega_{S} \otimes \mathcal{O}_{C}$ has a quotient isomorphic to the trivial sheaf $\mathcal{O}_{C}$. These curves are the obstruction to the ampleness of $\Omega_{S}$. For example, a smooth curve of genus 1 on $S$ is a non-ample curve.

Let $T_{S}=\Omega_{S}^{*}$ be the tangent sheaf and let $\pi: \mathbb{P}\left(T_{S}\right) \rightarrow S$ be the projection. As $\pi_{*} \mathcal{O}_{\mathbb{P}\left(T_{S}\right)}(1)=\Omega_{S}$ and the cotangent sheaf is generated by its global sections, we can define a map:

$$
\psi: \mathbb{P}\left(T_{S}\right) \rightarrow \mathbb{P}\left(H^{0}\left(\Omega_{S}\right)^{*}\right)=\mathbb{P}^{q-1}
$$

called the cotangent map of $S$, such that $\mathcal{O}_{\mathbb{P}\left(T_{S}\right)}(1)=\psi^{*}\left(\mathcal{O}_{\mathbb{P} q-1}(1)\right)$.

X. Roulleau: Graduate School of Mathematical Sciences, The University of Tokyo, 3-8-1 Komaba, Meguro, Tokyo 153-8914, Japan. e-mail: roulleau@ms.u-tokyo.ac.jp

Mathematics Subject Classification (2000): Primary 14J29; Secondary 14J45, 14J50, 14J70, $32 \mathrm{G} 20$ 
This map is the object of the paper [10]. In the present paper, we study the cotangent map and the configurations of the non-ample curves on Fano surfaces.

These surfaces were discovered by Fano and interest in them has been stimulated by the works of Clemens and Griffiths [4] Tyurin [12], [13] in 1971. By definition, a Fano surface is the Hilbert scheme of lines of a smooth cubic threefold $F \hookrightarrow \mathbb{P}^{4}$. This scheme is a surface $S$ that verifies Hypothesis 2 and has irregularity $q=5$.

By [4, Tangent Bundle Theorem 12.37], the image of the cotangent map $\psi$ : $\mathbb{P}\left(T_{S}\right) \rightarrow \mathbb{P}\left(H^{0}\left(\Omega_{S}\right)^{*}\right)$ of $S$ is a hypersurface $F^{\prime}$ of $\mathbb{P}\left(H^{0}\left(\Omega_{S}\right)^{*}\right) \simeq \mathbb{P}^{4}$ that is isomorphic to the original cubic $F$. Moreover, when we identify $F$ and $F^{\prime}$, the triple $\left(\mathbb{P}\left(T_{S}\right), \pi, \psi\right)$ is the universal family of lines on $F$.

The main results presented here are:

Theorem 3. (A) The non-ample curves of a Fano surface $S$ are the smooth curves of genus 1.

(B) There are only 10 configurations of smooth curves of genus 1 on the Fano surfaces. We know a plane model of each curve, the number of these curves and their intersection numbers.

(C) For each Fano surface $S$, we construct a particular sub-group $\mathbf{G}_{S}$ of its automorphism group. This construction goes in such a way that the knowledge of the group $\mathbf{G}_{S}$ gives the knowledge of the elliptic curve configurations on $S$ and reciprocally, the knowledge of the elliptic curve configuration on $S$ determines the group $\mathbf{G}_{S}$.

The results are summarized in the Classification Theorem 26. The number $n_{S}$ of elliptic curves on a Fano surface $S$ verifies $0 \leq n_{S} \leq 30$. The surfaces for which $n_{S}>0$ constitute a seven-dimensional family in the 10 dimensional moduli space of Fano surfaces.

The Picard number $\rho_{S}$ of a Fano surface $S$ satisfies $1 \leq \rho_{S} \leq 25$ and we can prove that it is 1 for $S$ generic. In fact, the number of elliptic curves is linked with the Picard number $\rho_{S}$ : we have $\rho_{S} \geq n_{S}$ unless $n_{S}=30$, in which case $\rho_{S}=25$. The number $n_{S}$ is also the number of one-dimensional fibres of the cotangent map $\psi$. This shows that the geometric properties of $\psi$ and the ampleness of the cotangent bundle vary non-trivially with the Fano surface.

We remark also that the results (A) and (B) of Theorem 3 are analogous to the classical statement on the canonical bundle of a minimal surface of general type which is ample if and only if the surface does not contain a (-2)-curve and the classification of canonical surfaces singularities.

We end this paper by an application of our study of Fano surfaces to construct cubic threefold whose intermediate Jacobian is isomorphic to a product of elliptic curves as an Abelian variety. The interest of this results is that in order to prove that the cubic threefolds are not rational, Clemens and Griffiths use the fact that their intermediate Jacobian cannot be isomorphic to a product of Jacobians of curves as a principally polarized Abelian variety.

Part of this paper was written at the Max-Plank Institute of Bonn, which is gratefully acknowledged. 


\section{Properties of the Fano surfaces and of their cotangent map}

\subsection{Properties of the cotangent map}

Let $S$ be a surface which verifies Hypothesis 2. In the introduction, we defined the cotangent map

$$
\psi: \mathbb{P}\left(T_{S}\right) \rightarrow \mathbb{P}\left(H^{0}\left(\Omega_{S}\right)^{*}\right)=\mathbb{P}^{q-1}
$$

by the surjective morphism $H^{0}\left(\Omega_{S}\right) \otimes O_{\mathbb{P}\left(T_{S}\right)} \rightarrow O_{\mathbb{P}\left(T_{S}\right)}(1)$. Here, we state general results about this morphism which will be used in the sequel; a complete treatment can be found in [10].

Let us recall that $\pi: \mathbb{P}\left(T_{S}\right) \rightarrow S$ is the projection. Let $s$ be a point of $S$. The restriction of the invertible sheaf $\mathcal{O}_{\mathbb{P}\left(T_{S}\right)}(1)$ to the fibre $\pi^{-1}(s) \simeq \mathbb{P}^{1}$ is the degree 1 invertible sheaf and the image under $\psi$ of that fibre is a line; we denote this line by $L_{s} \hookrightarrow \mathbb{P}^{q-1}$.

Let $G(2, q)=G\left(2, H^{0}\left(\Omega_{S}\right)^{*}\right)$ be the Grassmannian of projective lines in $\mathbb{P}^{q-1}$. By the universal property of the Grassmannian, the cotangent map induces a map

$$
\mathcal{G}: S \rightarrow G(2, q)
$$

called the Gauss map of $S$. The image by $\mathcal{G}$ of a point $s$ in $S$ is the line $L_{S}$.

Let $A$ be the Albanese variety $S$; its tangent space at 0 is $H^{0}\left(\Omega_{S}\right)^{*}$.

Proposition 4. Let $C \hookrightarrow S$ be a curve. The image under $\psi$ of $\pi^{-1}(C)$ is a cone if and only if $C$ is a non-ample curve.

An elliptic curve $E \hookrightarrow S$ is a non-ample curve. Let $p_{E}$ be the vertex of the cone $\psi\left(\pi^{-1}(E)\right)$. The underlying space of the point $p_{E}$ is the tangent space to the elliptic curve $\vartheta(E)$ translated in 0 .

Proof. The first assertion is [10, lemme 2.1].

Let $E$ be an elliptic curve on $S$. The natural quotient $\Omega_{S} \otimes \mathcal{O}_{E} \rightarrow \Omega_{E}$ is a trivial quotient, hence $E$ is non-ample. The last assertion results from the definition of the cotangent map.

Proposition 5. [10, cor. 2.12] Let $C \hookrightarrow S$ be a non-ample curve on a surface with irregularity $q>4$. Suppose that the vertex $p$ of the cone $T=\psi\left(\pi^{-1}(C)\right)$ is a smooth point of the image of $\psi$. Then $T$ is contained in the projective tangent space at $p$ of $F \hookrightarrow \mathbb{P}^{q-1}$ and one of the two following possibilities occurs:

(a) $C^{2}<0$ and $C$ is a smooth curve of genus 1.

(b) $C^{2}=0$ and an integral multiple of $C$ is a fiber of a fibration $f: S \rightarrow B$ onto a curve of genus $b$ with $q-3 \leq b \leq q-2$.

Let $C \hookrightarrow S$ be a curve and let $K$ be a canonical divisor of $\mathrm{S}$.

Proposition 6. [10, prop. 1.20] The degree of the cycle $\psi_{*} \pi^{*} C$ equals $K C$. 


\subsection{Main properties of Fano surfaces}

We recall here some properties of Fano surfaces needed in the sequel. The main references are the works of Clemens and Griffiths [4] and of Tyurin [12].

Let $F$ be a smooth cubic hypersurface of $\mathbb{P}^{4}$ and let $S$ be its Fano surface of lines. This surface verifies Hypothesis 2 and has irregularity $q=5$. Moreover:

Theorem 7. [4, Tangent Bundle Theorem 12.37] The image of the cotangent map of $S$ is a cubic hypersurface $F^{\prime} \hookrightarrow \mathbb{P}\left(H^{0}\left(\Omega_{S}\right)^{*}\right) \simeq \mathbb{P}^{4}$ isomorphic to the cubic $F \hookrightarrow \mathbb{P}^{4}$. Under the identification of $H^{0}\left(\Omega_{S}\right)^{*}$ and $H^{0}\left(\mathbb{P}^{4}, \mathcal{O}_{\mathbb{P}^{4}}(1)\right)$, and $F^{\prime}$ and $F$, the triple $\left(\mathbb{P}\left(T_{S}\right), \pi, \psi\right)$ is the universal family of lines of $F$.

The Chern numbers of a Fano surface verify : $c_{1}^{2}=45, c_{2}=27$. The cotangent map has degree 6: there are 6 lines through a generic point of $F$.

Lemma 8. [4, Thm. 12.37], [2, Cor. Parag. 4] The Gauss map and the Albanese map are embeddings.

Let $s$ be a point of $S$ and let $C_{s}$ be the closure of points $t \neq s$ in $S$ such that the line $L_{t}$ intersects the line $L_{s}$.

Proposition 9. [4, Parag. 10] The incidence divisor $C_{s}$ is ample, has self-intersection $C_{s}^{2}=5$ and arithmetical genus 11 . The divisor $3 C_{s}$ is numerically equivalent to a canonical divisor.

\subsection{Properties of a non-ample curve on a Fano surface}

Let $S$ be a Fano surface and let $F \hookrightarrow \mathbb{P}^{4}$ be the image of its cotangent map $\psi$. For a point $p$ of $F$, we denote by $T_{F, p} \hookrightarrow \mathbb{P}^{4}$ the projective tangent hyperplane to $F$ at $p$. For $E \hookrightarrow S$ a non-ample curve, we denote by $p_{E}$ the vertex of the cone $\psi\left(\pi^{-1}(E)\right)$.

Proposition 10. A curve $E$ on $S$ is non-ample if and only if it is an elliptic curve. Let $E \hookrightarrow S$ be elliptic curve. The cone $\psi\left(\pi^{-1}(E)\right)$ is the section of $F$ by the hyperplane $T_{F, p_{E}}$, furthermore : $E^{2}=-3, C_{S} E=1$.

Proof. Let $E \hookrightarrow S$ be a non-ample curve. By Proposition 5, the intersection of $F$ by $T_{F, p_{E}}$ contains the cone $\psi\left(\pi^{-1}(E)\right)$. As a smooth cubic threefold does not contain a plane, the hyperplane section of $F$ by $T_{F, p_{E}}$ is irreducible and is the cone $\psi\left(\pi^{-1}(E)\right)$. This cone has degree 3 . As the Gauss map is an embedding, the restriction of $\psi$ on $\pi^{-1}(E)$ is birational onto its image, thus $\psi_{*} \pi^{*} E=\psi\left(\pi^{-1}(E)\right)$ and Proposition 6 implies that

$$
K E=\operatorname{deg} \psi_{*} \pi^{*} E=3,
$$

where $K$ is a canonical divisor of $S$.

By Proposition 5, the curve $E$ satisfies $E^{2} \leq 0$. The number $2 p_{a}(E)-2=$ $E^{2}+K E=E^{2}+3$ must be divisible by 2 , hence $E^{2} \neq 0$. Proposition 5 imply that $E$ is an elliptic curve, thus: $E^{2}=-3$. Since $K$ is numerically equivalent to $3 C_{s}$, we obtain: $E C_{s}=1$.

The following proposition is [4, Parag. 8, 10]:

Proposition 11. A Fano surface contains at most 30 smooth curves of genus 1 . 


\subsection{The automorphism groups of the cubic and of the Fano surface}

Let us denote by $\operatorname{Aut}(X)$ the automorphisms group of a variety $X$. Let $S$ be a Fano surface and let $F$ be the image of the cotangent map of $S$.

An element $h \in \operatorname{Aut}(F)$ preserves the lines and by the Tangent Bundle Theorem 7, it acts on $S$ by an element denoted by $\rho(h)$.

Let be $\tau \in \operatorname{Aut}(S)$. The automorphism $\tau$ acts on the space $H^{0}\left(\Omega_{S}\right)^{*}$ by an automorphism denoted by $\tau^{*} \in G L\left(H^{0}\left(\Omega_{S}\right)^{*}\right)$. We remark also that $\tau$ induce an automorphism of the Albanese variety of $S:$ the morphism $\tau^{*}$ is the differential of that automorphism.

We denote by $\tilde{\tau} \in P G L\left(H^{0}\left(\Omega_{S}\right)^{*}\right)$ the projectivisation of $\tau^{*}$.

The following proposition is an immediate consequence of the definitions and the Tangent Bundle Theorem 7 ; we skip its proof because of its length.

Proposition 12. The morphism $\rho: \operatorname{Aut}(F) \rightarrow \operatorname{Aut}(S)$ is an isomorphism and its inverse is the morphism $\operatorname{Aut}(S) \rightarrow \operatorname{Aut}(F) ; \tau \rightarrow \widetilde{\tau}$.

In particular, for a point $s$ of $S$ and $\tau \in \operatorname{Aut}(S)$, we have $: L_{\tau s}=\widetilde{\tau}\left(L_{S}\right)$.

\section{Configurations of the elliptic curves}

\subsection{Configurations of 2 or 3 elliptic curves}

For two sub-varieties $V_{1}, V_{2}$ of $\mathbb{P}^{4}$, we denote by $\left\langle V_{1}, V_{2}\right\rangle$ their linear hull.

Let $E \hookrightarrow S$ be an elliptic curve on the Fano surface $S$ and let $p_{E}$ be the vertex of the cone $\psi\left(\pi^{-1}(E)\right)$ : this cone is the intersection of $F$ and $T_{F, p_{E}}$.

Let $s$ be a point of $S$ outside the curve $E$ : the line $L_{S}$ corresponding to the point $s$ is not inside the cone $\psi\left(\pi^{-1}(E)\right)$ and $X_{s}:=\left\langle L_{s}, p_{E}\right\rangle$ is a plane. This plane cuts the cubic $F$ in three lines:

(1) the line $L_{S}$,

(2) the line $L_{\gamma_{E} s}$ (on the cone) through the vertex $p_{E}$ and the intersection point of $L_{S}$ and the hyperplane $T_{F, p_{E}}$,

(3) the residual line $L_{\sigma_{E} s}$ such that:

$$
X_{s} F=L_{s}+L_{\gamma_{E} s}+L_{\sigma_{E} s} .
$$

As an Albanese morphism of $S$ is an embedding, the surface $S$ does not contain a rational curve ; furthermore $E$ has genus $>0$. Then [6, Cor. 1.44] implies that the rational maps $\sigma_{E}: S \rightarrow S$ and $\gamma_{E}: S \rightarrow E$ are everywhere defined. As the plane $X_{S}(s$ in $S \backslash E)$ is equal to $X_{\sigma_{E} s}$, the morphism $\sigma_{E}^{2}$ is the identity on $S \backslash E$, thus $\sigma_{E}$ is an involutive automorphism.

Let $s, t$ be two points of the curve $E \hookrightarrow S$. The line $L_{s}$ cuts the line $L_{t}$ at the vertex of the cone $\psi\left(\pi^{-1}(E)\right)$ : thus the point $s$ lies on the incidence divisor $C_{t}$ and there exists a residual divisor $R_{t}$ such that:

$$
C_{t}=E+R_{t}
$$


Theorem 13. (a) Let $t$ be a point of $E \hookrightarrow S$. The divisor $R_{t}$ is the fibre at $t$ of $\gamma_{E}$ and has arithmetical genus 7. The morphisms $\gamma_{E}$ and $\sigma_{E}$ satisfy $\gamma_{E} \sigma_{E}=\gamma_{E}$.

(b) The automorphism $-\sigma_{E}^{*} \in G L\left(H^{o}\left(\Omega_{S}\right)^{*}\right)$ is a complex order 2 reflection. The eigenspace of $\sigma_{E}^{*}$ with eigenvalue 1 is the underlying space of the vertex $p_{E} \in \mathbb{P}^{4}$.

(c) Let $E^{\prime} \hookrightarrow S$ be an elliptic curve, distinct from $E$. Then $0 \leq E E^{\prime} \leq 1$.

(d) The automorphisms $\sigma_{E}$ and $\sigma_{E^{\prime}}$ verify:

$$
\left(\sigma_{E} \sigma_{E^{\prime}}\right)^{3-E E^{\prime}}=I d_{S} .
$$

(e) If $E E^{\prime}=1$, then the fibration $\gamma_{E}$ contracts $E^{\prime}$.

(f) If $E E^{\prime}=0$, then there exists a third elliptic curve $E^{\prime \prime}$ such that

$$
\sigma_{E}\left(E^{\prime}\right)=\sigma_{E^{\prime}}(E)=E^{\prime \prime}
$$

Moreover the curves $E^{\prime}$ and $E^{\prime \prime}$ are sections of $\gamma_{E}$.

Let us prove Theorem 13. Let $t$ be a point of $E \hookrightarrow S$. Let $s$ be a generic point of $R_{t}=C_{t}-E$. The line $L_{s}$ cuts the line $L_{t} \hookrightarrow \psi\left(\pi^{-1}(E)\right)$ in a point different from $p_{E}$ and by definition: $\gamma_{E} s=t$, thus $s$ is a point of $\gamma_{E}^{-1}(t)$. This proves that $R_{t}$ is a component of $\gamma_{E}^{-1}(t)$. Conversely, we have $R_{t}^{2}=\left(C_{t}-E\right)^{2}=5-2+(-3)=0$, by [1, Chap. III, Zariski's Lemma 8.2], that implies that $R_{t}$ is the fibre at $t$ of $\gamma_{E}$.

A canonical divisor $K$ is numerically equivalent to $3 C_{t}$, hence $K R_{t}=K\left(C_{t}-\right.$ $E)=12$ and $R_{t}$ has arithmetical genus $\frac{12+0}{2}+1$.

The plane $X_{S}(s$ in $S \backslash E)$ is equal to the plane $X_{\sigma_{E} s}$, hence $\gamma_{E} s=\gamma_{E} \sigma_{E} s$.

Let $E^{\prime} \hookrightarrow S$ be an elliptic curve. As $R_{t}$ is a fibre, we have:

$$
R_{t} E^{\prime}=\left(C_{t}-E\right) E^{\prime}=1-E E^{\prime} \geq 0 .
$$

If $E=E^{\prime}$, we see that $\gamma_{E}$ has degree 4 on $E$. Suppose now that $E \neq E^{\prime}$, then $E E^{\prime} \geq 0$ and hence $0 \leq E E^{\prime} \leq 1$. We proved (a) and (c).

If $E E^{\prime}=1$, then $R_{t} E=0$ and $E^{\prime}$ is contained in a fibre of $\gamma_{E}$, hence (e). If $E E^{\prime}=0$, then $R_{t} E^{\prime}=1$, thus $E^{\prime}$ is a section of $\gamma_{E}$.

Let us prove the property (b). Up to a variable change, we can suppose that the vertex $p_{E}$ of the cone $\psi\left(\pi^{-1}(E)\right)$ is the point $(1: 0: 0: 0: 0)$. It is easy to prove that, up to a variable change, an equation of $F$ is:

$$
F=\left\{x_{1}^{2} x_{2}+G\left(x_{2}, x_{3}, x_{4}, x_{5}\right)=0\right\} .
$$

The automorphism $h: x \rightarrow\left(-x_{1}: x_{2}: x_{3}: x_{4}: x_{5}\right)$ acts on $F$. The geometric interpretation of $h$ is given as follows: if $q$ is a generic point of $F$, then the points $p, q, h(q)$ lie on a line. Let $s$ be a generic point of $S$. It is then easy to check that the lines $L_{S}, h\left(L_{S}\right)$ and the point $p_{E}$ span a plane : this is thus the plane $X_{S}$ and we see that $h\left(L_{S}\right)=L_{\sigma_{E} s}$, thus : $h=\widetilde{\sigma_{E}}$ (see the notations of Theorem 12).

The automorphism $\widetilde{\sigma_{E}}=h$ fix the point $p_{E}$ and the hyperplane $\left\{x_{1}=0\right\} \hookrightarrow \mathbb{P}^{4}$. The fixed locus of $\sigma_{E}$ is thus the union of $E$ and the 27 points corresponding to the lines in the intersection of $F$ and the hyperplane $\left\{x_{1}=0\right\}$ (as we can verify this intersection is a smooth cubic surface). 
By Proposition 12, the automorphism $\sigma_{E}^{*} \in G L\left(H^{0}\left(\Omega_{S}\right)^{*}\right)$ is equal to:

$$
g: x \rightarrow\left(-x_{1}, x_{2}, x_{3}, x_{4}, x_{5}\right)
$$

or to $-g$. Let $\vartheta: S \rightarrow A$ be an Albanese morphism. As $\sigma_{E}$ is the identity on $E$, the automorphism $\sigma_{E}^{*} \in G L\left(H^{0}\left(\Omega_{S}\right)^{*}\right)$ (which is the differential of the action of $\sigma_{E}$ on $A$ ) is the identity on the tangent space of the curve $\vartheta(E)$ translated at 0 . By Proposition 4 , this space is $\mathbb{C}(1,0,0,0,0) \subset H^{0}\left(\Omega_{S}\right)^{*}$. Thus $\sigma_{E}^{*}=-g$ and $-\sigma_{E}^{*}$ is a complex reflection of order 2 . We proved $b$ ).

Let $E^{\prime} \neq E$ a second smooth curve of genus 1 on $S$. Let us prove that $\left(\sigma_{E} \sigma_{E^{\prime}}\right)^{3-E E^{\prime}}=I d_{S}$.

Case $E E^{\prime}=1$. Suppose that $E E^{\prime}=1$. A generic hyperplane section $Y$ of the cone $\psi\left(\pi^{-1}\left(E^{\prime}\right)\right)$ parameterizes the lines of this cone and is a plane model of $E^{\prime}$ : we will identify $Y$ and $E^{\prime}$. Let $t$ be the intersection point of $E$ and $E^{\prime}$ : with this neutral element, the curve $E^{\prime}$ is an elliptic curve.

Let $s$ be a point of $E^{\prime}$ different from $t$, then by definition of $\sigma_{E}$ and $\gamma_{E}$ :

$$
X_{E, s} F=L_{s}+L_{\gamma_{E} s}+L_{\sigma_{E} s}
$$

where $X_{E, s}:=\left\langle L_{s}, p_{E}\right\rangle$. Since the line $L_{s}$ cuts the line $L_{t}$ at the vertex $p_{E^{\prime}}$, we have $X_{E, s}=\left\langle L_{s}, L_{t}\right\rangle$. Thus the point $t$ is one of the three points $s, \gamma_{E} s, \sigma_{E} s$. As $s \neq t$ and the automorphism $\sigma_{E}$ preserves $E$, the point $\sigma_{E} s$ is not an element of $E$, hence $\gamma_{E} s=t$. Thus $E^{\prime}$ is a component of $\gamma_{E}^{-1}(t)$.

The plane $X_{E, s}$ contains the lines $L_{s}, L_{t}$ that are in the hyperplane section $\psi\left(\pi^{-1}\left(E^{\prime}\right)\right)$ of $F$, thus the third line $L_{\sigma_{E} S}$ is also in the cone $\psi\left(\pi^{-1}\left(E^{\prime}\right)\right)$ and the point $\sigma_{E} s$ is on $E^{\prime}$. Because of the relation

$$
X_{E, s} F=L_{s}+L_{\gamma_{E} s}+L_{\sigma_{E} s},
$$

the three points $s, t=\gamma_{E} s, \sigma_{E} s$ are on a line in the plane model $Y$, hence $\sigma_{E} s=-s$ for all points $s$ of $E^{\prime}$.

Remark 14. Since $\sigma_{E}(s)=-s$ on $E^{\prime}$, the endomorphism $\sigma_{E}^{*} \in G L\left(H^{0}\left(\Omega_{S}\right)^{*}\right)$ is the morphism of multiplication by -1 on the tangent space to the curve $\vartheta\left(E^{\prime}\right)$ (translated to 0). Thus : $\widetilde{\sigma_{E}} p_{E^{\prime}}=p_{E^{\prime}}$ and $p_{E^{\prime}}$ is the intersection point of the line $L_{t}$ and the hyperplane of fixed points of $\widetilde{\sigma_{E}}$. This implies that the points $p_{E}$ and $p_{E^{\prime}}$ are the only vertices of cones on the line $L_{t}$.

Let $s$ be a generic point of $S$, then

$$
\widetilde{\sigma_{E^{\prime}}} X_{E, s}=\widetilde{\sigma_{E^{\prime}}}\left\langle p_{E}, L_{s}\right\rangle=\left\langle p_{E}, L_{\sigma_{E^{\prime}}}\right\rangle=X_{E, \sigma_{E^{\prime}} s},
$$

hence:

$$
\widetilde{\sigma_{E^{\prime}}} X_{E, s} F=L_{\sigma_{E^{\prime}}}+L_{\gamma_{E} \sigma_{E^{\prime}} s}+L_{\sigma_{E} \sigma_{E^{\prime}}} .
$$

But $X_{E, s} F=L_{s}+L_{\gamma_{E} s}+L_{\sigma_{E} s}$ hence:

$$
\widetilde{\sigma_{E^{\prime}}} X_{E, s} F=L_{\sigma_{E^{\prime}} s}+L_{\sigma_{E^{\prime}} \gamma_{E} s}+L_{\sigma_{E^{\prime}} \sigma_{E} s} .
$$


Since $\gamma_{E} \sigma_{E^{\prime}} s$ and $\sigma_{E^{\prime}} \gamma_{E} s$ are points of $E$, we see that $\sigma_{E} \sigma_{E^{\prime}} s=\sigma_{E^{\prime}} \sigma_{E} s$, thus $\left(\sigma_{E} \sigma_{E^{\prime}}\right)^{2}=I d_{S}$.

Case $E E^{\prime}=0$. Suppose now that $E E^{\prime}=0$. Let $s$ be a point of $E^{\prime}$. By definition $X_{E, s}=\left\langle p_{E}, L_{s}\right\rangle$ and:

$$
X_{E, s} F=L_{s}+L_{\gamma_{E} s}+L_{\sigma_{E} s} .
$$

Suppose that $\sigma_{E} s$ is a point of $E^{\prime}$, then plane $X_{E, s}$ cuts the cone $\psi\left(\pi^{-1}\left(E^{\prime}\right)\right)$ into two lines : $L_{s}$ and $L_{\sigma_{E} s}$. As this cone has degree 3 , the intersection of $X_{E, s}$ and $\psi\left(\pi^{-1}\left(E^{\prime}\right)\right)$ contains the third line $L_{\gamma_{E} s}$. Since $\psi\left(\pi^{-1}\left(E^{\prime}\right)\right)$ is a cone, that implies that $\gamma_{E} s$ is a point of $E^{\prime}$ : this is a impossible because $E E^{\prime}=0$. Thus $\sigma_{E} s$ is not a point of $E^{\prime}$.

The automorphism $\sigma_{E}$ fixes $E$. Hence the point $\sigma_{E} S$ is not a point of $E$. This proves that the surface $S$ contains a third smooth curve $E^{\prime \prime}=\sigma_{E}\left(E^{\prime}\right)$ of genus 1 and that $E E^{\prime \prime}=E^{\prime} E^{\prime \prime}=0$.

For a point $s$ of $E^{\prime}$, the plane $X_{E, s}=\left\langle p_{E}, L_{s}\right\rangle$ contains the line $L_{\gamma_{E} s}$ and the point $p_{E^{\prime}} \in L_{S}$, hence:

$$
X_{E, s}=\left\langle p_{E}, L_{s}\right\rangle=\left\langle p_{E^{\prime}}, L_{\gamma_{E} s}\right\rangle=X_{E^{\prime}, \gamma_{E} s} .
$$

But we have

$$
X_{E, s} F=L_{s}+L_{\gamma_{E} s}+L_{\sigma_{E} s}
$$

and

$$
X_{E^{\prime}, \gamma_{E} s} F=L_{\gamma_{E} s}+L_{\gamma_{E^{\prime} \gamma_{E} s}}+L_{\sigma_{E^{\prime} \gamma_{E} s}} .
$$

Since the points $s, \gamma_{E} s$ and $\sigma_{E} s$ are respectively points of $E^{\prime}, E$ and $E^{\prime \prime}$, we see that for all points $s$ of $E^{\prime}$ :

$$
\sigma_{E^{\prime}} \gamma_{E} s=\sigma_{E} s
$$

Hence the restriction of $\sigma_{E^{\prime}}$ to $E$ is a morphism from $E$ to $E^{\prime \prime}$ and $\sigma_{E^{\prime}}(E)=$ $\sigma_{E}\left(E^{\prime}\right)=E^{\prime \prime}$, this proves f).

Let $s$ be a point of $E^{\prime}$. The lines $L_{s}, L_{\gamma_{E} s}$ and $L_{\sigma_{E} s}$ contain respectively the vertices $p_{E^{\prime}}, p_{E}$, and $p_{E^{\prime \prime}}$. As $s$ varies in $E^{\prime}$, the plane $X_{E, s}$ varies and the linear hull $\ell$ of the points $p_{E}, p_{E^{\prime}}$ and $p_{E^{\prime \prime}}$ cannot be a plane: it is a line.

Remark 15. The line $\ell$ lies outside the cubic $F$, otherwise the curves $E$ and $E^{\prime}$ would have a common point. The points $p_{E}, p_{E^{\prime}}$ and $p_{E^{\prime \prime}}$ are the intersection points of $\ell$ and the cubic $F$.

Let $s$ be a point of $S$. The morphism $\widetilde{\sigma}_{E^{\prime}}$ verifies : $\widetilde{\sigma}_{E^{\prime}}\left(L_{S}\right)=L_{\sigma_{E^{\prime}}}$, and furthermore:

$$
\widetilde{\sigma}_{E^{\prime}} X_{E, \sigma_{E^{\prime}}}=\widetilde{\sigma}_{E^{\prime}}\left\langle p_{E}, L_{\sigma_{E^{\prime}}}\right\rangle=\left\langle p_{E^{\prime \prime}}, L_{s}\right\rangle=X_{E^{\prime \prime}, s} .
$$

We have $X_{E, \sigma_{E^{\prime}} s} F=L_{\sigma_{E^{\prime}} s}+L_{\gamma_{E} \sigma_{E^{\prime}} s}+L_{\sigma_{E} \sigma_{E^{\prime}}}$. Hence

$$
\widetilde{\sigma}_{E^{\prime}} X_{E, \sigma_{E^{\prime}} s} F=L_{\sigma_{E^{\prime}}^{2} s}+L_{\sigma_{E^{\prime}} \gamma_{E} \sigma_{E^{\prime}} s}+L_{\sigma_{E^{\prime}} \sigma_{E} \sigma_{E^{\prime}} s},
$$


but by 3.1:

$$
\widetilde{\sigma}_{E^{\prime}} X_{E, \sigma_{E^{\prime}}} F=X_{E^{\prime \prime}, s} F
$$

Since $X_{E^{\prime \prime}, s} F=L_{s}+L_{\gamma_{E^{\prime \prime}} s}+L_{\sigma_{E^{\prime \prime}} s}$, we see that $\sigma_{E^{\prime}} \sigma_{E} \sigma_{E^{\prime}}=\sigma_{E^{\prime \prime}}$. So the group generated by $\sigma_{E}, \sigma_{E^{\prime}}, \sigma_{E^{\prime \prime}}$ is isomorphic to $\Sigma_{3}$ and $\left(\sigma_{E} \sigma_{E^{\prime}}\right)^{3}=I d_{S}$.

This ends the proof of Theorem 13.

Let us now study the configuration of three elliptic curves.

Proposition 16. Let $E_{1}, E_{2}$ and $E$ be three elliptic curves on $S$ such that $E_{1} E=$ $E_{2} E=1$. Then: $E_{1} E_{2}=0$ and the curve $E_{3}=\sigma_{E_{1}}\left(E_{2}\right)$ verifies $E_{3} E=1$.

Proof. Suppose $E_{1} E_{2}=1$. This implies that the line through $p_{E_{1}}$ and $p_{E_{2}}$ lies on the cone $\psi\left(\pi^{-1}(E)\right)$ and hence goes through $p_{E}$. But by Remark 14, the three points $p_{E}, p_{E_{1}}$ and $p_{E_{2}}$ cannot be on a line. Hence $E_{1} E_{2}=0$.

Since $E E_{1}=1$, we have $\sigma_{E_{1}}(E)=E$ and $E_{3} E=\sigma_{E_{1}}\left(E_{2}\right) \sigma_{E_{1}}(E)=E_{2}$ $E=1$.

\subsection{The graph of the configuration of the vertices of cones}

Let $\mathcal{E}$ be the set of elliptic curves on $S$. Let us consider the following graph $\mathbb{G}$ : the set of vertices of $\mathbb{G}$ is $\mathcal{E}$ and an edge links $E \in \mathcal{E}$ to $E^{\prime} \in \mathcal{E}$ if and only if $E E^{\prime}=0$. Let $\mathbf{G}_{S}$ be the sub-group of $\operatorname{Aut}(S)$ generated by the automorphisms $\sigma_{E}, E \in \mathcal{E}$. We have the three following relations between its generators:

(a) for all $E \in \mathcal{E}, \sigma_{E}$ verifies $\sigma_{E}^{2}=I d_{S}$,

(b) an edge links $E$ and $E^{\prime}$ if and only if $\left(\sigma_{E} \sigma_{E^{\prime}}\right)^{3}=I d_{S}$,

(c) otherwise $\left(\sigma_{E} \sigma_{E^{\prime}}\right)^{2}=I d_{S}$.

The following corollary is a consequence of Proposition 16.

Corollary 17. Let $E_{1}, E_{2}$ and $E_{3}$ be three elements of $\mathcal{E}$. At least one edge links two of the three vertices $E_{1}, E_{2}, E_{3}$ of the graph $\mathbb{G}$.

There is no sub-group of $\mathbf{G}_{S}$ generated by some elements $\sigma_{E}, E \in \mathcal{E}$ and isomorphic to $(\mathbb{Z} / 2 \mathbb{Z})^{3}$.

Proof. If there are no edges between the vertices $E_{1}$ and $E_{3}$ and between the vertices $E_{2}$ and $E_{3}$, then $E_{1} E_{3}=E_{2} E_{3}=1$ and the Proposition 16 implies that $E_{1} E_{2}=0$. Thus an edge links the vertices $E_{1}$ and $E_{2}$. The second assertion is a reformulation of the first.

We remark that if the graph $\mathbb{G}$ has $m$ connected components, then the group $\mathbf{G}_{S}$ is the direct product of $m$ sub-groups. The Corollary 17 can be reformulated as follows:

Corollary 18. The graph $\mathbb{G}$ is connected or $\mathbb{G}$ has two connected components $\mathbb{G}_{1}, \mathbb{G}_{2}$ such that two different vertices of a component $\mathbb{G}_{i}$ are linked by an edge. 
Let $\mathcal{E}^{\prime}$ be a sub-set of $\mathcal{E}$. Let $\mathbb{G}^{\prime}$ be the graph whose set of vertices is $\mathcal{E}^{\prime}$ and such that an edge links two elements of $\mathcal{E}^{\prime}$ if and only if these vertices are linked by an edge in $\mathbb{G}$. Suppose that the three relations (a), (b) and (c) above are the only ones between the elements $\sigma_{E}, E \in \mathcal{E}^{\prime}$. The group generated by the automorphisms $\sigma_{E}, E \in \mathcal{E}^{\prime}$ is then a Weyl group, which we denote by $\mathbf{G}_{S}^{\prime}$.

Corollary 19. If the graph $\mathbb{G}^{\prime}$ is connected and has $n$ vertices, then $1 \leq n \leq 4$ and the group $\mathbf{G}_{S}^{\prime}$ is the permutation group of the set of $n+1$ elements.

Proof. By corollary 17 and the classification of the Weyl groups [9], the graph $\mathbb{G}^{\prime}$ must be one of the graphs $A_{n}, 1 \leq n \leq 4$. The Weyl group $W\left(A_{n}\right)$ associated to $A_{n}$ is the permutation group of $n+1$ elements.

\subsection{Restrictions on the complex reflection groups}

Let us denote by $G_{S}$ the complex reflection group generated by the order 2 reflections $-\sigma_{E}^{*} \in G L\left(H^{0}\left(\Omega_{S}\right)^{*}\right), E \hookrightarrow S$ elliptic curve. For the basic properties of reflection groups see $[5,11]$ or [7], here we recall some them:

Definition 20. A reflection in a space $V$ is a linear transformation of $V$ of finite order with fixed point set an hyperplane of $V$. A reflection group is a finite group generated by reflections.

Let $G_{1}$ and $G_{2}$ be two reflection groups acting on spaces $V_{1}$ and $V_{2}$, we say (improperly) that $G_{1}$ is a reflection sub-group of $G_{2}$, if there exists an injective morphism $G_{1} \rightarrow G_{2}$ of complex reflection groups. In this case, we denote the elements of $G_{1}$ and $G_{2}$ by the same letters.

The list of the 37 types of irreducible reflection groups was compiled by Shephard and Todd [11].

Let us fix some notation. Let $m, n>0$ be integers and let $p$ be an integer dividing $m$. We denote by $\Sigma_{n} \subset G L_{n}(\mathbb{C})$ the group of permutation matrices and by $A(m, p, n) \subset G L_{n}(\mathbb{C})$ the group of diagonal matrices $D$ of order $m$ such that $\operatorname{det}(D)^{\frac{m}{p}}=1$. The type 2 reflection groups are the groups $G(m, p, n)$ generated by $A(m, p, n)$ and $\Sigma_{n}$.

The type 3 groups constitute the family [ $]^{n}$ for $n \in \mathbb{N} \backslash\{0,1\}$ where the [ $]^{n}$ is the group of morphisms $\mathbb{C} \rightarrow \mathbb{C} ; x \rightarrow \xi x, \xi^{n}=1$.

Theorem 21. An irreducible sub-group of $G_{S}$ generated by reflections of order 2 is isomorphic to one of the following groups:

$$
\{1\},[]^{2}, G(3,3, n), G(1,1, n)=\Sigma_{n}, 2 \leq n \leq 5 .
$$

Proof. By the Remarks 14 and 15, a projective line of $\mathbb{P}^{4}$ contains at most 3 vertices of a cone, hence a 2 dimensional reflection sub-group of $G_{S}$ contains at most 3 reflections of order 2 . The groups of type 4 to 22 are 2 dimensional irreducible groups. They either have no or at least 6 reflections of order 2 [5, Table p. 395] : none of these groups can be a reflection sub-group of $G_{S}$. 
The groups numbered 25, 29, 31, 32, 33, 34 possess either no or at least 40 reflections of order 2 [5, p. 412]. Since reflections of order 2 of $G_{S}$ are in bijection with elliptic curves on $S$ and a Fano surface contains at most 30 elliptic curves (Proposition 11), none of these groups is a sub-group of $G_{S}$.

The groups 23, 28, 30, 35, 36, 37 are real reflection groups [11, p. 299] which have been excluded by the Corollary 19 .

The group 26 has a reflection sub-group isomorphic to the group number 4 already excluded [11, p. 302].

The groups 24 and 27 have two reflections $R_{1}$ and $R_{2}$ of order 2 such that $\left(R_{1} R_{2}\right)^{4}=1$ [11, p. 299] and hence they cannot be sub-groups of $G_{S}$.

It thus remains to study the reflection groups of types 1,2 and 3 .

Let $M$ be an irreducible sub-group of $G_{S}$ generated by order 2 reflections.

Type 3. It is immediate that there exists $n>1$ such that []$^{n} \simeq M$ if and only if $n=2$.

Type 2 and 1 . The group $G(m, p, n)$ (where $p$ divides $m \in \mathbb{N}^{*}$ and $n>1$ ) is an $n$ dimensional irreducible reflection group if and only if $m>1$ and $(m, p, n) \neq$ $(2,2,2)$. The representation $G(1,1, n)=\Sigma_{n}$ of the permutation group breaks up into a 1 dimensional trivial representation and an $n-1$ dimensional irreducible representation $W\left(A_{n-1}\right)$ called the standard representation. The groups:

$$
W\left(A_{n}\right), \quad n \in \mathbb{N}^{*}
$$

constitute the number 1 reflection type in the Shephard-Todd classification.

Let $m, p, n$ be integers such that $m>0, p$ divides $m$ and $n>1$. Suppose that $M$ is the group $G(m, p, n)$. Theorem 13 implies that a group generated by two reflections of order 2 of $G_{S}$ is the diedral group of order 4 or 6 . For $m>1$, the group $G(m, p, n)$ contains a diedral sub-group of order $2 m$ generated by two reflections of order 2. Thus the integer $m$ is an element of $\{1,2,3\}$; moreover $n \leq 5$.

The group $G(2,2,2)$ is not irreducible and is thus excluded. The groups $G(2,1, n)$ with $n \geq 3$ and the groups $G(2,2, n)$ with $n \geq 4$ contain sub-groups isomorphic to $(\mathbb{Z} / 2 \mathbb{Z})^{3}$ generated by reflections of order 2 . By Corollary 18 , such groups cannot be reflection sub-groups of $G_{S}$.

The reflection group $\Sigma_{4}$ is isomorphic to the group $G(2,2,3)$ plus the trivial representation. So $G(2,2,3)$ is implicitly in the list of Theorem 21 .

The group $G(3,1, n)$ cannot be isomorphic to $M$ because its order 2 reflections generate the strict sub-group $G(3,3, n) \subset G(3,1, n)$.

By Corollary 19, if $W\left(A_{n}\right)$ is a reflection sub-group of $G_{S}$, then $n \leq 4$. The group $\Sigma_{n}$ is isomorphic to the reflection group $W\left(A_{n-1}\right)$ plus the trivial representation.

Hence, we proved that $M$ is isomorphic to one of the groups $\{1\},[]^{2}, G(3,3, n)$, $G(1,1, n)=\Sigma_{n}, 2 \leq n \leq 5$.

\subsection{Classification of groups and Fano surfaces}

Let us classify the Fano surfaces according to the configuration of their elliptic curves. Let us recall the notations : to each elliptic curve $E \hookrightarrow S$ corresponds 
an automorphism $\sigma_{E}$ of $S$ and the group $G_{S}$ is generated by the elements $-\sigma_{E}^{*} \in$ $G L\left(H^{0}\left(\Omega_{S}\right)^{*}\right)$, where the automorphisms $\sigma_{E}^{*}$ are defined in paragraph 2.4. We will use the following remark:

Remark 22. Let $F_{e q}$ be an equation of the image $F$ of the cotangent map of a Fano surface $S$. There exists a morphism:

$$
\chi: G_{S} \rightarrow \mathbb{C}^{*}
$$

such that $F_{e q} \circ N=\chi(N) F_{e q}$ for all $N \in G_{S}$.

Thus we are looking for cubic forms $F_{e q}$, reflection groups $G$ and morphisms $\chi: G \rightarrow \mathbb{C}^{*}$ such that $F_{e q} \circ N=\chi(N) F_{e q}$ for all $N \in G$ and such that $\left\{F_{e q}=0\right\}$ is smooth.

We need some notations and preliminary materials.

The order of $G(m, p, n)$ is equal to $\frac{m}{p} m^{n-1} n !$ and the number of its order 2 reflections is $m \frac{n(n-1)}{2}$. The group $G(m, m, n)$ acts on the polynomial space of $\mathbb{C}^{n}$. The algebra of invariant polynomials is generated by the polynomials:

$$
\sum_{i=1}^{i=n} x_{i}^{m k}, \quad k \in\{1, \ldots, n-1\}
$$

and by $x_{1} x_{2} \ldots x_{n}$ (see [11]).

Let $S$ be a Fano surface such that the group $G(m, m, n)(m \in\{1,3\}, 2 \leq n \leq 5)$ is a reflection sub-group of $G_{S}$. Let be $m \in\{1,3\}$ and $n>1$. We easily check that the only non-trivial morphism from $G(m, m, n)$ to $\mathbb{C}^{*}$ is the determinant. We call a polynomial $P$ an anti-invariant of $G(m, m, n)$ if $P \circ N=(\operatorname{det} N) P$ for all $N \in G(m, m, n)$. We verify that:

Lemma 23. The only reflection groups $G(m, m, n)$ with $m \in\{1,3\}$ and $n \geq 2$ that possess an anti-invariant polynomial of degree $\leq 3$ are $G(1,1,2), G(3,3,2)$ and $G(1,1,3)$.

Notation 24. For $\lambda^{3} \neq 1$, we denote by $E_{\lambda}$ the smooth plane cubic: $x^{3}+y^{3}+z^{3}-$ $3 \lambda x y z=0$.

We denote by $A$ the Albanese variety of $S$, by $\vartheta: S \rightarrow A$ an Albanese morphism, by $e_{1}, \ldots, e_{5}$ the dual basis of the basis $x_{1}, \ldots, x_{5} \in H^{o}\left(\Omega_{S}\right)$. If $v \in$ $H^{0}\left(\Omega_{S}\right)^{*}$ is a non zero vector, $\mathbb{C} v$ is the vector space generated by $v$ or the point of $\mathbb{P}^{4}=\mathbb{P}\left(H^{0}\left(\Omega_{S}\right)^{*}\right)$ corresponding to this space, we specify as need be. We denote by $\mu_{3}$ the group of third roots of unity.

Recall (see Theorem 13 and its proof) that there is a one to one correspondence between:

(a) the elliptic curves $E$ on $S$.

(b) the order 2 reflections $R=-\sigma_{E}^{*}$ of $G_{S}$.

(c) the cones $\psi\left(\pi^{-1}(E)\right)$ on $F$.

(d) the vertices $p_{E}$ of the cones $\psi\left(\pi^{-1}(E)\right) \hookrightarrow F$. 
When we consider an order 2 reflection $R$ corresponding to an elliptic curve $E \hookrightarrow S$, the vertex $p_{E}$ of the cone $\psi\left(\pi^{-1}(E)\right)$ is the point of $\mathbb{P}^{4}$ corresponding to the eigenspace with eigenvalue -1 of $R \in G L\left(H^{0}\left(\Omega_{S}\right)^{*}\right)=G L_{5}(\mathbb{C})$.

The cone $\psi\left(\pi^{-1}(E)\right)$ is the intersection of the cubic $F$ and the tangent space at $p_{E}$. A plane model of the curve $E$ is the intersection of this cone with the hyperplane of fixed points of $\widetilde{\sigma_{E}}$ (where $\widetilde{\sigma_{E}}$ is the projectivization of $R$ ).

In order to know the intersection number between two elliptic curves $E_{1}$ and $E_{2}$ corresponding to the reflections $R_{1}$ and $R_{2}$, it suffice to compute the order ( $=2$ or 3 ) of the element $R_{1} R_{2}$ and to use the formula:

$$
\left(R_{1} R_{2}\right)^{3-E_{1} E_{2}}=I d .
$$

It can also be verified by hand if the line between the vertices $p_{E_{1}}$ and $p_{E_{2}}$ is or not inside the cubic, accordingly: $E_{1} E_{2}=1$ or 0 .

In the sequel, we proceed to the classification of the elliptic curve configurations on Fano surfaces according to the dimension of the studied irreducible reflection sub-group of $G_{S}$; this group must be in the list of Theorem 21 .

- 1 dimensional sub-group of $G_{S}$.

We proved in paragraph 3.1, that when $G_{S}$ contains the group [ $]^{2}$ generated by a reflection $R$, the cubic can be written as follows:

$$
F=\left\{x_{1}^{2} x_{2}+G\left(x_{2}, \ldots, x_{5}\right)=0\right\}
$$

where $G$ is a cubic form and $R$ acts by $x \rightarrow\left(-x_{1}, x_{2}, x_{3}, x_{4}, x_{5}\right)$. The tangent space at $p=(1: 0: 0: 0: 0)$ is $T_{F, p}=\left\{x_{2}=0\right\}$. This point $p$ is the vertex of a cone in $F$. This cone is the intersection of $T_{F, p}$ and $F$. Let us denote by $E \hookrightarrow S$ the elliptic curve on the Fano surface that parameterizes the lines on the cone.

The hyperplane $\left\{x_{1}=0\right\}$ is an invariant of the cubic because it is the fixed locus of $\widetilde{\sigma_{E}}$. A plane model of $E$ is obtained by the intersection of $F$ and the plane $\left\{x_{1}=x_{2}=0\right\}$.

We see in that way, that given a pair $(T, E)$ where $T$ is a smooth cubic surface (say given by $T=\left\{G\left(x_{2}, \ldots, x_{5}\right)=0\right\}$ ) and $E$ is a smooth hyperplane section of $T$ (say $E=T \cap\left\{x_{2}=0\right\}$ ), we can associate the pair $\left(S, E^{\prime}\right)$ where $S$ is the Fano surface of $\left\{x_{1}^{2} x_{2}+G\left(x_{2}, \ldots, x_{5}\right)=0\right\}$ and $E^{\prime}$ is an elliptic curve on it isomorphic to $E$. Reciprocally, given the pair $\left(S, E^{\prime}\right)$, we can recover the cubic $F$ and the pair $(T, E)$, up to isomorphism. As the moduli space of isomorphism class of such pairs $(T, E)$ is 7 dimensional, the moduli of Fano surfaces that contains an elliptic curve is a 7 dimensional sub-space of the 10 dimensional moduli of Fano surfaces.

- 2 dimensional reflection sub-groups of $G_{S}$.

- The anti-invariant polynomials of the reflection group $G(3,3,2)$ yield singular cubics. The invariants of the group $G(3,3,2)$ are generated by the polynomials:

$$
x_{1}^{3}+x_{2}^{3}, x_{1} x_{2}, x_{3}, x_{4}, x_{5} .
$$

Up to a change of variables, the cubic $F$ is:

$$
F=\left\{x_{1}^{3}+x_{2}^{3}+3 x_{1} x_{2} l\left(x_{3}, x_{4}, x_{5}\right)+x_{3}^{3}+x_{4}^{3}+x_{5}^{3}-3 \lambda x_{3} x_{4} x_{5}=0\right\}
$$

where $l$ is a linear form and $\lambda \in \mathbb{C}$. 
The 3 points $\mathbb{C}\left(e_{1}-\beta e_{2}\right), \beta \in \mu_{3}$ (see Notations 24$)$ are vertices of cones. The Fano surface $S$ contains three disjoint elliptic curves $E_{12}^{\beta}, \beta \in \mu_{3}$ that have the following model:

$$
\left\{x_{3}^{3}+x_{4}^{3}+x_{5}^{3}-3 \lambda x_{3} x_{4} x_{5}+l^{3}=0\right\} .
$$

Note that we also have studied the reflection group $G(1,1,3)=\Sigma_{3}$ because its representation decomposes into the trivial one plus the standard representation $W\left(A_{2}\right)$ which is equal to $G(3,3,2)$.

- 3 dimensional reflection sub-groups of $G_{S}$.

- The invariants of $G(3,3,3)$ are generated by:

$$
x_{1}^{3}+x_{2}^{3}+x_{3}^{3}, x_{1} x_{2} x_{3}, x_{4}, x_{5}
$$

Up to a variables change, there exist coordinates there exists $\lambda \in \mathbb{C}$ such that:

$$
F=\left\{x_{1}^{3}+x_{2}^{3}+x_{3}^{3}-3 \lambda x_{1} x_{2} x_{3}+x_{4}^{3}+x_{5}^{3}=0\right\} .
$$

The group $G(3,3,3) \times G(3,3,2)$ is a reflection sub-group of $G_{S}$. The 9 points $\mathbb{C}\left(e_{i}-\beta e_{j}\right), 1 \leq i<j \leq 3, \beta \in \mu_{3}$ are vertices of cones. The corresponding elliptic curves:

$$
E_{i j}^{\beta}, 1 \leq i<j \leq 3, \quad \beta \in \mu_{3}
$$

on the Fano surface $S$ are isomorphic to the curve $E_{0}$ and are disjoint.

The 3 points $\mathbb{C}\left(e_{4}-\beta e_{5}\right), \beta \in \mu_{3}$ are vertices of cones. The corresponding 3 elliptic curves $E_{45}^{\beta}, \beta \in \mu_{3}$ are disjoint and isomorphic to the curve $E_{\lambda}$. For all $1 \leq i<j \leq 3,(\beta, \gamma) \in \mu_{3}^{2}$, we have:

$$
E_{i j}^{\beta} E_{45}^{\gamma}=1
$$

The Fano surface contains 12 elliptic curves.

- The invariants of degree less than or equal to 3 of $\Sigma_{4}$ are generated by:

$$
x_{5}^{k}, x_{1}^{k}+x_{2}^{k}+x_{3}^{k}+x_{4}^{k}, \quad k \in\{0,1,2,3\} .
$$

Let $F$ be a smooth cubic defined by an element of the 6 dimensional space of invariant cubics. For $1 \leq i<j \leq 4$, the point $\mathbb{C}\left(e_{i}-e_{j}\right)$ of $\mathbb{P}^{4}$ is the vertex of a cone on $F$; let us denote by:

$$
E_{i j} \hookrightarrow S
$$

the corresponding elliptic curve on the Fano surface $S$. We have:

$$
E_{i j} E_{s t}= \begin{cases}1 & \text { if }\{i, j\} \cap\{s, t\}=\emptyset \\ -3 & \text { if } E_{i j}=E_{s t} \\ 0 & \text { otherwise. }\end{cases}
$$

The surface $S$ contains 6 elliptic curves. 
- 4 dimensional reflection sub-groups of $G_{S}$.

- Suppose that $\Sigma_{5}$ is a reflection sub-group of $G_{S}$. The invariants of degree less than or equal to 3 of $\Sigma_{4}$ are generated by:

$$
x_{1}^{k}+x_{2}^{k}+x_{3}^{k}+x_{4}^{k}+x_{5}^{k}, \quad k \in\{0,1,2,3\} .
$$

There exist $\lambda, \mu \in \mathbb{C}$ such that the image of the cotangent map of $S$ is:

$$
F=\left\{\sum_{i=1}^{i=5} x_{i}^{3}+\lambda\left(\sum_{i=1}^{i=5} x_{i}\right)\left(\sum_{i=1}^{i=5} x_{i}^{2}\right)+\mu\left(\sum_{i=1}^{i=5} x_{i}\right)^{3}=0\right\} .
$$

For $1 \leq i<j \leq 5$, the point

$$
p_{i j}=\mathbb{C}\left(e_{i}-e_{j}\right)
$$

is the vertex of a cone and we denote by $E_{i j} \hookrightarrow S$ the corresponding elliptic curve. We have:

$$
E_{i j} E_{s t}= \begin{cases}1 & \text { if }\{i, j\} \cap\{s, t\}=\emptyset \\ -3 & \text { if } E_{i j}=E_{s t} \\ 0 & \text { otherwise. }\end{cases}
$$

The dual graph of this configuration of 10 elliptic curves is the trivalent Petersen graph.

- 5 dimensional reflection sub-group of $G_{S}$.

- The Fermat cubic:

$$
F=\left\{x_{1}^{3}+x_{2}^{3}+x_{3}^{3}+x_{4}^{3}+x_{5}^{3}=0\right\}
$$

is, up to isomorphism, the only cubic stable under $G(3,3,4)$ and $G(3,3,5)$. The points $\mathbb{C}\left(e_{i}-\beta e_{j}\right), 1 \leq i<j \leq 5, \beta \in \mu_{3}$ are vertices of cones on $F$. Its Fano surface $S$ is the unique Fano surface that contains 30 smooth curves of genus 1 . These curves are numbered:

$$
E_{i j}^{\beta}, 1 \leq i<j \leq 5, \beta \in \mu_{3} .
$$

Let $E_{i j}^{\gamma}$ and $E_{s t}^{\beta}$ be two such curves, then:

$$
E_{i j}^{\beta} E_{s t}^{\gamma}= \begin{cases}1 & \text { if }\{i, j\} \cap\{s, t\}=\emptyset \\ -3 & \text { if } E_{i j}^{\beta}=E_{s t}^{\gamma} \\ 0 & \text { otherwise. }\end{cases}
$$

A remarkable property of this surface is that its Néron-Severi group $N S(S)$ has rank $25=\operatorname{dim} H^{1}\left(S, \Omega_{S}\right)$ and $N S(S) \otimes \mathbb{Q}$ is generated by the 30 elliptic curves.

Now, we study the case for which the reflection group $G_{S}$ is not irreducible. Corollary 18 proves that: 
Lemma 25. If the reflection group $G_{S}$ is not irreducible, it is the direct product of two irreducible reflection groups $\mathbb{W}_{1}$ and $\mathbb{W}_{2}$ such that if $R_{1}$ and $R_{2}$ are two different reflections of order 2 of the group $\mathbb{W}_{i}$, we have:

$$
\left(R_{1} R_{2}\right)^{3}=I d .
$$

The groups with this last property and listed in Theorem 21 are:

$$
[]^{2}, G(3,3,2) \text { or } G(3,3,3) \text {. }
$$

- The case where one of the groups $\mathbb{W}_{i}(i \in\{1,2\})$ of Lemma 25 is equal to $G(3,3,3)$ has already been studied. In that case

$$
\mathbb{W}_{1} \times \mathbb{W}_{2} \simeq G(3,3,3) \times G(3,3,2)
$$

is a reflection sub-group of $G_{S}$.

- If []$^{2} \times[]^{2}$ is a reflection sub-group of $G_{S}$, there exist coordinates such that:

$$
F=\left\{x_{1}^{2} l_{1}\left(x_{3}, x_{4}, x_{5}\right)+x_{2}^{2} l_{2}\left(x_{3}, x_{4}, x_{5}\right)+x_{3}^{3}+x_{4}^{3}+x_{5}^{3}-3 \lambda x_{3} x_{4} x_{5}=0\right\}
$$

where $l_{1}$ and $l_{2}$ are two linearly independent forms. The points $\mathbb{C} e_{1}$ and $\mathbb{C} e_{2}$ are vertices of cones on $F$. The Fano surface $S$ contains two elliptic curves $E, E^{\prime}$ such that $E E^{\prime}=1$.

- If $G(3,3,2) \times[]^{2}$ is a reflection sub-group of $G_{S}$, then there exist coordinates such that:

$$
F=\left\{x_{1}^{3}+x_{2}^{3}-3 \lambda x_{1} x_{2} l_{1}\left(x_{4}, x_{5}\right)+x_{3}^{2} l_{2}\left(x_{4}, x_{5}\right)+x_{4}^{3}+x_{5}^{3}=0\right\},
$$

where $l_{1}$ are $l_{2}$ linear forms. The points $\mathbb{C}\left(e_{1}-\beta e_{2}\right), \beta \in \mu_{3}$ and $\mathbb{C} e_{3}$ are vertices of cones. The Fano surface contains three disjoint elliptic curves that cut another elliptic curve. The dual graph of this configuration is the graph $D_{4}$.

- The last case is the group $G(3,3,2) \times G(3,3,2)$ for which the cubic is:

$$
F=\left\{x_{1}^{3}+x_{2}^{3}+3 a x_{1} x_{2} x_{5}+x_{3}^{3}+x_{4}^{3}+3 b x_{3} x_{4} x_{5}+x_{5}^{3}=0\right\},
$$

$(a, b \in \mathbb{C})$. The points $\mathbb{C}\left(e_{1}-\beta e_{2}\right), \mathbb{C}\left(e_{3}-\beta e_{4}\right), \beta \in \mu_{3}$ are vertices of cones on $F$. The Fano surface $S$ contains 3 disjoint elliptic curves $E_{12}^{\beta}, \beta \in \mu_{3}$ isomorphic to the plane cubic

$$
\left\{x_{3}^{3}+x_{4}^{3}+\left(1+a^{3}\right) x_{5}^{3}+3 b x_{3} x_{4} x_{5}=0\right\},
$$

and three others disjoint elliptic curves $E_{34}^{\alpha}, \alpha \in \mu_{3}$ isomorphic to the cubic:

$$
\left\{x_{1}^{3}+x_{2}^{3}+\left(1+b^{3}\right) x_{5}^{3}+3 a x_{1} x_{2} x_{5}=0\right\} .
$$

These curves verify $E_{12}^{\beta} E_{34}^{\gamma}=1\left(\beta, \gamma \in \mu_{3}\right)$. The surface $S$ contains 6 elliptic curves.

Recall that for each elliptic curve $E \hookrightarrow S$, we denoted by $\sigma_{E}: S \rightarrow S$ the involution associated to $E$ and that $G_{S}$ is a reflection group that is isomorphic to the group $\mathbf{G}_{S}$ generated by the automorphisms $\sigma_{E}$. Let us denote by $n_{S}$ the number of elliptic curves on $S$. The following theorem gives the configuration classification of elliptic curves on Fano surfaces: 
Theorem 26. (Classification Theorem). If the group $G_{S}$ is irreducible, then it is isomorphic to one of the following groups:

\begin{tabular}{|c|c|c|c|c|c|c|}
\hline Group $G_{S}$ & $\{1\}$ & {[]$^{2}$} & $G(3,3,2)$ & $\Sigma_{4}$ & $\Sigma_{5}$ & $G(3,3,5)$ \\
\hline$n_{S}$ & 0 & 1 & 3 & 6 & 10 & 30 \\
\hline Order of $G_{S}$ & 1 & 2 & 6 & 24 & 120 & 9,720 \\
\hline
\end{tabular}

Otherwise, $G_{S}$ is isomorphic to one of the following groups:

\begin{tabular}{|c|c|c|c|}
\hline[]$^{2} \times[]^{2}$ & $G(3,3,2) \times[]^{2}$ & $G(3,3,2) \times G(3,3,2)$ & $G(3,3,3) \times G(3,3,2)$ \\
\hline 2 & 4 & 6 & 12 \\
\hline 4 & 12 & 36 & 324 \\
\hline
\end{tabular}

Let $E, E^{\prime}$ be two elliptic curves on $S$. We know the intersection number $E E^{\prime}$ and a plane model of $E$.

\section{Intermediate Jacobians isomorphic to a product of elliptic curves}

Let $\lambda \in \mathbb{C}, \lambda^{3} \neq 1$. The Fano surface $S_{\lambda}$ of the cubic

$$
F_{\lambda}=\left\{x_{1}^{3}+x_{2}^{3}+x_{3}^{3}-3 \lambda x_{1} x_{2} x_{3}+x_{4}^{3}+x_{5}^{3}\right\} \hookrightarrow \mathbb{P}^{4}
$$

possesses 12 smooth curves of genus 1 for which we use the notations of the previous paragraph. Let $\alpha$ be a primitive third root of unity. Let $\lambda \in \mathbb{C}$ be such that the elliptic curve $E_{\lambda}=\left\{x_{1}^{3}+x_{2}^{3}+x_{3}^{3}-3 \lambda x_{1} x_{2} x_{3}=0\right\}$ has complex multiplication by $\mathbb{Q}(\alpha)$. Let $A_{\lambda}$ be the Albanese variety of the Fano surface $S_{\lambda}$ of $F_{\lambda}$ and let $\vartheta: S_{\lambda} \rightarrow A_{\lambda}$ be an Albanese map. In this last section, we prove the following result:

Theorem 27. The Abelian variety $A_{\lambda}$ is isomorphic to a product of elliptic curves.

Let $S$ be a Fano surface. The Albanese map $\vartheta: S \rightarrow A$ is an embedding and we consider points of $S$ as points of $A$.

Lemma 28. [4, (11.9)] There is a point $u_{o}$ on A such that for all points $s_{1}, s_{2}, s_{3}$ on $S$ such that the lines $L_{S_{1}}, L_{S_{2}}, L_{S_{3}}$ are coplanar, we have:

$$
s_{1}+s_{2}+s_{3}=u_{o} .
$$

Let $E$ be an elliptic curve on a Fano surface $S$. In order to avoid heavy notations, we consider points of $E$ as points of $A$ and the morphism of Abelian varieties are taken modulo translation : the differential and degree of such class is well defined. For the points $s$ on $S$, the lines $L_{S}, L_{\gamma_{E} S}, L_{\sigma_{E} S}$ are coplanar, hence the morphism $s \rightarrow s+\sigma_{E} s+\gamma_{E} s$ is constant i.e. its differential is 0 . Let $I_{A}$ be the identity of $A$, let

$$
\Gamma_{E}: A \rightarrow E, \Sigma_{E}: A \rightarrow A
$$

be the morphisms such that $\Gamma_{E} \circ \vartheta=\gamma_{E}$ and $\Sigma_{E} \circ \vartheta=\vartheta \circ \sigma_{E}$. The differential of $\Sigma_{E}$ is $\sigma_{E}^{*}$. The morphism $I d_{A}+\Sigma_{E}+\Gamma_{E}$ is constant, hence: 
Lemma 29. The differential of $\Gamma_{E}$ is the endomorphism $N_{E} \in \operatorname{End}\left(H^{o}\left(\Omega_{S}\right)^{*}\right)$ such that:

$$
I d+\sigma_{E}^{*}+N_{E}=0
$$

where I is the identity of $H^{o}\left(\Omega_{S}\right)^{*}$.

Let us take $S=S_{\lambda}$. We denote by $\Gamma_{i j}^{\beta}: A_{\lambda} \rightarrow E_{i j}^{\beta}$ the morphism such that $\Gamma_{i j}^{\beta} \circ \vartheta=\gamma_{E_{i j}^{\beta}}$.

Lemma 30. The degree of the morphism

$$
\Gamma=\left(\Gamma_{12}^{1}, \Gamma_{23}^{1}, \Gamma_{12}^{\alpha}, \Gamma_{45}^{1}, \Gamma_{45}^{\alpha}\right): A_{\lambda} \rightarrow E_{12}^{1} \times E_{23}^{1} \times E_{12}^{\alpha} \times E_{45}^{1} \times E_{45}^{\alpha}
$$

divides 81 .

Proof. Let $\Upsilon$ be the morphism $\Gamma$ composed with the natural morphism $E_{12}^{1} \times E_{23}^{1} \times$ $E_{12}^{\alpha} \times E_{45}^{1} \times E_{45}^{\alpha} \rightarrow A_{\lambda}$. By Lemma 29, we can compute the differential $d \Upsilon$ of $\Upsilon$ and we find $|\operatorname{det}(d \Upsilon)|^{2}=81$, hence the assertion.

We can now complete the proof of Theorem 27.

Proof. By Lemma 30 the Abelian variety $A_{\lambda}$ is isogenous to a product of elliptic curves ; by the choice of the parameter $\lambda$, these curves have complex multiplication by the same field : the Néron-Severi group of $A_{\lambda}$ has thus rank $25=h^{1,1}\left(A_{\lambda}\right)$. Then [3, Chap. 5, Exer. 5.6 (10)] imply that $A_{\lambda}$ is isomorphic to a product of elliptic curves.

\section{References}

[1] Barth, W., Hulek, K., Peters, C., Van De Ven, A.: Compact complex surfaces, volume 4 of Ergebnisse der Mathematik und ihrer Grenzgebiete, 2nd edn. Springer-Verlag, Berlin (2004)

[2] Beauville, A.: Les singularités du diviseur $\Theta$ de la jacobienne intermédiaire de la cubique dans $\mathbb{P}^{4}$ Algebraic threefolds (Proc. Varenna 1981), LNM 947, pp. 190-208. Springer, Berlin (1982)

[3] Birkenhake, C., Lange, H.: Complex Abelian varieties, volume 302 of Grundlehren der Mathematischen Wissenschaften, 2nd edn. Springer, Berlin (2004)

[4] Clemens, H., Griffiths, P.: The intermediate Jacobian of the cubic threefold. Ann. Math. 95, 281-356 (1972)

[5] Cohen, A.: Finite complex reflection groups. Ann. Scient. Ec. Norm. Sup. 4ème Sèrie 9, 379-436 (1976)

[6] Debarre, O.: Higher-dimensional Algebraic Geometry. Universitext. Springer, New York (2001)

[7] Dolgachev, I.: Reflection groups in algebraic geometry. Bull. Am. Math. Soc. (N.S.) 45(1), 1-60 (2008)

[8] Gieseker, D.: On a theorem of Bogomolov on Chern classes of stables bundle. Am. J. Math. 101, 77-85 (1979)

[9] Humphreys, J.: Reflection Groups and Coxeter Groups, Cambridge Studies in Advanced Mathematics, vol. 29. Cambridge University Press, Cambridge (1990) 
[10] Roulleau, X.: L'application cotangente des surfaces de type général. preprint Arxiv: 0902.4069, to be published in Geom. Dedicata

[11] Shephard, G.C., Todd, J.A.: Finite unitary reflection groups. Can. J. Math. 5, 364-383 (1953)

[12] Tyurin, A.N.: On the Fano surface of a nonsingular cubic in $\mathbb{P}^{4}$. Math. Ussr Izv. 4, 1207-1214 (1970)

[13] Tyurin, A.N.: The geometry of the Fano surface of a nonsingular cubic $F \subset \mathbb{P}^{4}$ and Torelli Theorems for Fano surfaces and cubics. Math. Ussr Izv. 5, 517-546 (1971) 